\title{
GCU
}

Glasgow Caledonian

University

University for the Common Good

\section{Enhancement of power transformer state of health diagnostics based on fuzzy logic system of DGA}

Aburaghiega, Ehnaish; Farrag, Mohamed Emad; Hepburn, Donald; Haggag, Ayman

Published in:

2018 Twentieth International Middle East Power Systems Conference (MEPCON)

DOI:

10.1109/MEPCON.2018.8635154

Publication date:

2019

Document Version

Author accepted manuscript

Link to publication in ResearchOnline

Citation for published version (Harvard):

Aburaghiega, E, Farrag, ME, Hepburn, D \& Haggag, A 2019, Enhancement of power transformer state of health diagnostics based on fuzzy logic system of DGA. in 2018 Twentieth International Middle East Power Systems Conference (MEPCON). 2018 20th International Middle East Power Systems Conference, MEPCON 2018 Proceedings, IEEE, pp. 400-405, 2018 Twentieth International Middle East Power Systems Conference (MEPCON), Cairo, Egypt, 18/12/18. https://doi.org/10.1109/MEPCON.2018.8635154

\section{General rights}

Copyright and moral rights for the publications made accessible in the public portal are retained by the authors and/or other copyright owners and it is a condition of accessing publications that users recognise and abide by the legal requirements associated with these rights.

Take down policy

If you believe that this document breaches copyright please view our takedown policy at https://edshare.gcu.ac.uk/id/eprint/5179 for details of how to contact us. 


\section{Enhancement of Power Transformer State of Health Diagnostics Based on Fuzzy Logic System of DGA}

\author{
Ehnaish Aburaghiega \\ School of Engineering and Built \\ Environment \\ Glasgow Caledonian University \\ Glasgow, UK \\ Ehnaish.Aburaghiega@gcu.ac.uk \\ Mohamed Emad Farrag \\ ${ }^{I}$ School of Engineering and Built \\ Environment \\ Glasgow Caledonian University \\ Glasgow, UK \\ ${ }^{2}$ Faculty of Industrial Education, \\ Helwan University, Egypt \\ Mohamed.Farrag@gcu.ac.uk
}

\author{
Donald Hepburn \\ School of Engineering and Built \\ Environment \\ Glasgow Caledonian University \\ Glasgow, UK \\ dmhepburn_gcu@yahoo.com
}

\author{
Ayman Haggag \\ Faculty of Industrial Education, \\ Helwan University, Egypt \\ haggag@techedu.helwan.edu.eg
}

\begin{abstract}
Dissolved Gas Analysis (DGA) of liquid insulation is an effective means for diagnosing power transformers. The gas contents in insulating oil can be gathered on-line and off-line to indicate the health condition of the transformers, thereafter there are many interpretations of the gas contents. In this work, Seven-fuzzy interpretation modules are individually established, tested and lately combined to monitor power transformers' health. The developed method incorporates trending of the concentration of the dissolved gases over the operating life. The approach processes current and/or historical DGA data, using the 7developed logic modules, to determine the current state of a transformer, provide information regarding the fault type, fault probability, fault severity and recommended future sampling interval in addition to operating procedure, consistent with industry standards. The developed diagnosis system has been validated using 1290 samples from fresh and previously tested mineral oil filled transformers. The proposed system is proved, based on field data, to be $99 \%$ accurate in identifying transformers being in normal or abnormal operation. For the cases where a transformer fault was known, the proposed technique has less than $2 \%$ inaccuracy in recognizing the fault's type in comparison to other approaches discussed in literature.
\end{abstract}

Keywords-Transformer diagnostics, On-line monitoring, fuzzy logic, DGA, Fault detection, Severity.

\section{INTRODUCTION}

Unscheduled loss of large transformer causes major disruptions in power supply, resulting in high financial penalties, in repair costs and environmental hazard [1]. Typically, mineral oil is the main medium to isolate the transformer components and providing the necessary cooling [2]. During normal operation, Hydrogen $\left(\mathrm{H}_{2}\right)$, Methane $\left(\mathrm{CH}_{4}\right)$, Acetylene $\left(\mathrm{C}_{2} \mathrm{H}_{2}\right)$, Ethylene $\left(\mathrm{C}_{2} \mathrm{H}_{4}\right)$, Ethane $\left(\mathrm{C}_{2} \mathrm{H}_{6}\right)$, Carbon monoxide $(\mathrm{CO})$ and Carbon Dioxide $\left(\mathrm{CO}_{2}\right)$ are released into the oil at low concentrations. Under fault conditions specific gases are generated at levels above that of normal operation [3]. Analysis of the gases in the oil, Dissolved Gas Analysis (DGA), gives information on transformer health, fault type and advice on future operation. Researchers have applied advanced algorithms, e.g. fuzzy logic systems [4], [5] neuro-fuzzy networks [6]-[8] and artificial neural networks [9]-[11], to improve the reliability of DGA interpretation and transformer fault diagnostics.

This contribution describes a fuzzy logic analysis system for monitoring power transformers based on combining several DGA interpretation methods to do the diagnosis. The developed method is capable of being used for both new and old transformers and incorporates trending of the concentration of the dissolved gases over the transformer's operating life. The proposed technique was validated in a large number of cases where the actual faults of the transformer were known and also on a wider set of DGA data where no information about the transformer condition was available. The trending of DGA was undertaken as well. The precision of the method is compared with the accuracy of other interpretation approaches included in the standards or proposed by other authors.

The proposed method, which can be used for off and on-line condition monitoring data, is divided into the following stages: (1) analyzing the gas concentration levels; (2) diagnosing the transformer health and identifying the fault type; (3) providing fault severity and sampling intervals for on-going processing; (4) recommending operating procedures for continuing operation. The system output offers information required by an operator, i.e. transformer health and what actions to take for unhealthy cases. The overall decision for a given transformer is automatically updated when gas concentrations are updated.

\section{Proposed ApProACH}

The proposed approach incorporates the development of fuzzy based analysis of most well-known DGA interpretation techniques, namely;

- IEEE standards for identification of transformer health condition [12].

- IEEE standards for determining operating procedure and sampling interval [12].

- Duval triangle 1 technique [13]

- Doernenburg ratio method [12].

- Key gas method [12].

A fuzzy module for each technique is developed including selection of membership functions, fuzzyfication, rule reasoning and defuzzification process. Due to space limitations, as creation of fuzzy systems is well established, the specifics of each module in the developed approach are not detailed here.

As shown in Figure 1, the diagnostic scheme starts by applying the IEEE-Filter, module 1, checks the 7-key gases and Total Dissolved Combustible Gasses (TDCG) levels to identify the Condition Status (C-S) of a transformer. If all gases are below the levels defined in the IEEE standard [12], the transformer is considered as operating satisfactorily (C-S is normal); if any of the gases exceeds their critical limit the transformer is reported as "abnormal".

Based on normal/abnormal decision, the second module, which is responsible for identifying the fault probability using TDCG level and output of the IEEE-Filter (normal/abnormal), is applied. Thereafter, modules 3-5 identify the fault type individually using fuzzy analysis of diagnostic techniques (Duval/Doernenburg/key gas), it is to be noted that Duval triangle 1 is used for the purpose of this study, as more recent developments of this technique yet depends on running triangle 1 first [14] 
It is acknowledged that DGA does not address all issues relating to power transformer management but it is used as a front line tool in assessing their condition.

The fault identified by each technique is then assessed in the combination module (module 6) to determine the most likely fault, based on the criteria that will be discussed in the following sections. Changes in TDCG levels between consecutive samples are also assessed and the output of the amalgamation of the three techniques and trending module (module 7) provides an overall indication of the transformer condition. The proposed approach can also be used to monitor transformers with no historical records of DGA available (designated as "new transformers" throughout the paper).

\section{A. The Propsed Approach for Intial Test on A Transformer}

This procedure analyses transformers which have not been tested previously. The seven gases of the current sample are applied to the process in Figure 1, as no previous DGA data is available to identify fault probability the data is applied without considering rate of change of gases. Fault probability is based on "IEEE-TDCG-level" algorithm and outputs Conditions 1 to 4 , the higher the TDCG level the higher the fault probability of course. The seven gases and the calculated TDCG are initially tested using the IEEE-Filter: if all gases and TDCG are below the specified level for normal operation then Doernenburg, Duval and Key gas techniques are disabled; the algorithm monitoring IEEE-TDCG-level will indicate a very low probability, i.e. Condition 1.

If any gas exceeds the limit, the transformer is marked as operating abnormally even if the TDCG level remains in Condition 1. If any gas exceeds its normal level and the TDCG level remains in condition 1, the fault probability will be low. As gases have exceeded limits, the module also examines the DGA data using the three monitoring techniques to determine fault type.

\section{B. System Operation for On-Going Transformer Monitoring}

For transformers which are already entered in a database, current and previous DGA data and dates are used. The new sample will be processed as discussed above for a new transformer but the previous samples' DGA data are used to determine the rate of gas production. TDCG for the two most recent samples and number of days between them are used to calculate the daily increment rate. The TDCG level of the new sample, the daily increment rate and the IEEE-Filter results feed into the Trending TDCG algorithm: rules for Trending TDCG analysis are based on the IEEE standard.

\section{Overall Decision of the Approach}

The approach initially determines if the current DGA sample has values indicating unsatisfactory condition. Thereafter the system provides information on the fault suspected, five stages are discussed as follows

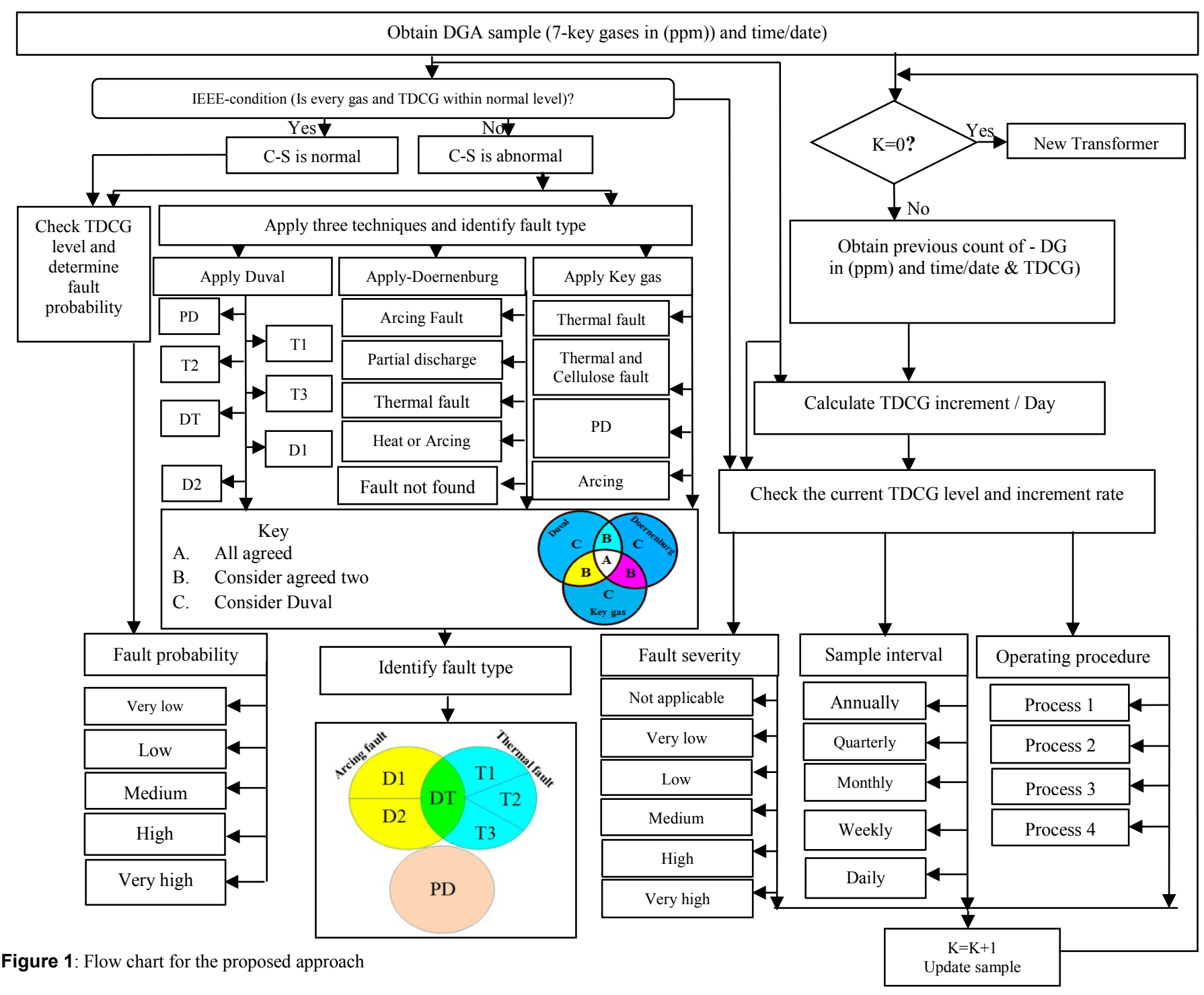




\section{1) Fault Type}

As shown in Figure 1, when no fault exists the system output will be Regular Operation (RO) indicator. Other faults are determined and each specified type of fault is indicated by the codes shown in Table I.

TABLE I. FAULT TYPE

\begin{tabular}{|c|c|c|}
\hline Code & Symbol & Fault \\
\hline 0 & RO & Regular Operation \\
\hline 1 & "PD" & PD \\
\hline 2 & "T1" & Excessive Heat $<300{ }^{\circ} \mathrm{C}$ \\
\hline 3 & "T2" & $300<$ Excessive Heat $<700{ }^{\circ} \mathrm{C}$ \\
\hline 4 & "T3" & Excessive Heat $>700{ }^{\circ} \mathrm{C}$ \\
\hline 5 & "DT" & Electrical and Heat \\
\hline 6 & "D1" & Low energy discharge \\
\hline 7 & "D2" & High energy Discharge \\
\hline 8 & "TF" & Excessive heat \\
\hline 9 & "AF" & Arcing fault \\
\hline
\end{tabular}

\section{2) Fault Probability}

According to [12], a fault probability can be assigned based on levels or ratios of the key-gases that are used to identify (C-S) of the transformer. The (C-S) and the TDCG are analyzed and, accordingly, transformer fault probability is classified as per the IEEE standards and as shown in Figure 1

\section{3) Fault Severity}

The fault severity can be assessed using the increment rate for each TDCG band. It should be noted that for data from a device classified as a new transformer this module remains "off" due to lack of previous DGA data. For a transformer which is indicated as healthy, the output of this module is "Not applicable". Processing data which includes previous DGA, the module's outputs for fault severity are shown in Figure 1.

\section{4) Sample Interval}

Based on the rate of change of TDCG and transformer condition the interval between tests can be determined. Recommendations, based on fault severity, can suggest sampling from annually to daily, as shown in Figure 1.

\section{5) Operation Procedures and Recommendation}

The sampling interval, TDCG level and increment rate are used to decide on the future operating procedure and provides advice on keeping a transformer working safely. The operating procedure must be followed, taking into account the sample intervals and other factors outlined above, as shown in Table II.

TABLE II. FAULT TYPE

\begin{tabular}{|c|c|c|}
\hline Code & Code meaning & Description \\
\hline 0 & Off & New transformer \\
\hline 1 & Operating procedure 1 & Continue normal operation \\
\hline 2 & Operating procedure 2 & $\begin{array}{c}\text { Exercise extreme caution. Analyze for } \\
\text { individual gases. Determine load } \\
\text { dependence }\end{array}$ \\
\hline 3 & Operating procedure 3 & $\begin{array}{c}\text { Exercise extreme Caution. Analyze for } \\
\text { individual gases, Plan outage. Advise } \\
\text { Manufacturer }\end{array}$ \\
\hline 4 & Operating procedure 4 & Consider removal from service \\
\hline
\end{tabular}

\section{MATLAB SCHEMATIC OF APPROACH}

As has been discussed, the overall assessment of the transformer condition has four output codes that indicate: fault type, fault probability, recommended sampling interval and operating procedure.

The developed fuzzy interpretation modules for the included DGA interpretation techniques are then integrated in one system implemented in Matlab. Figure 2 shows the approach's outputs for a specific case that was used during validation of the diagnostic system.

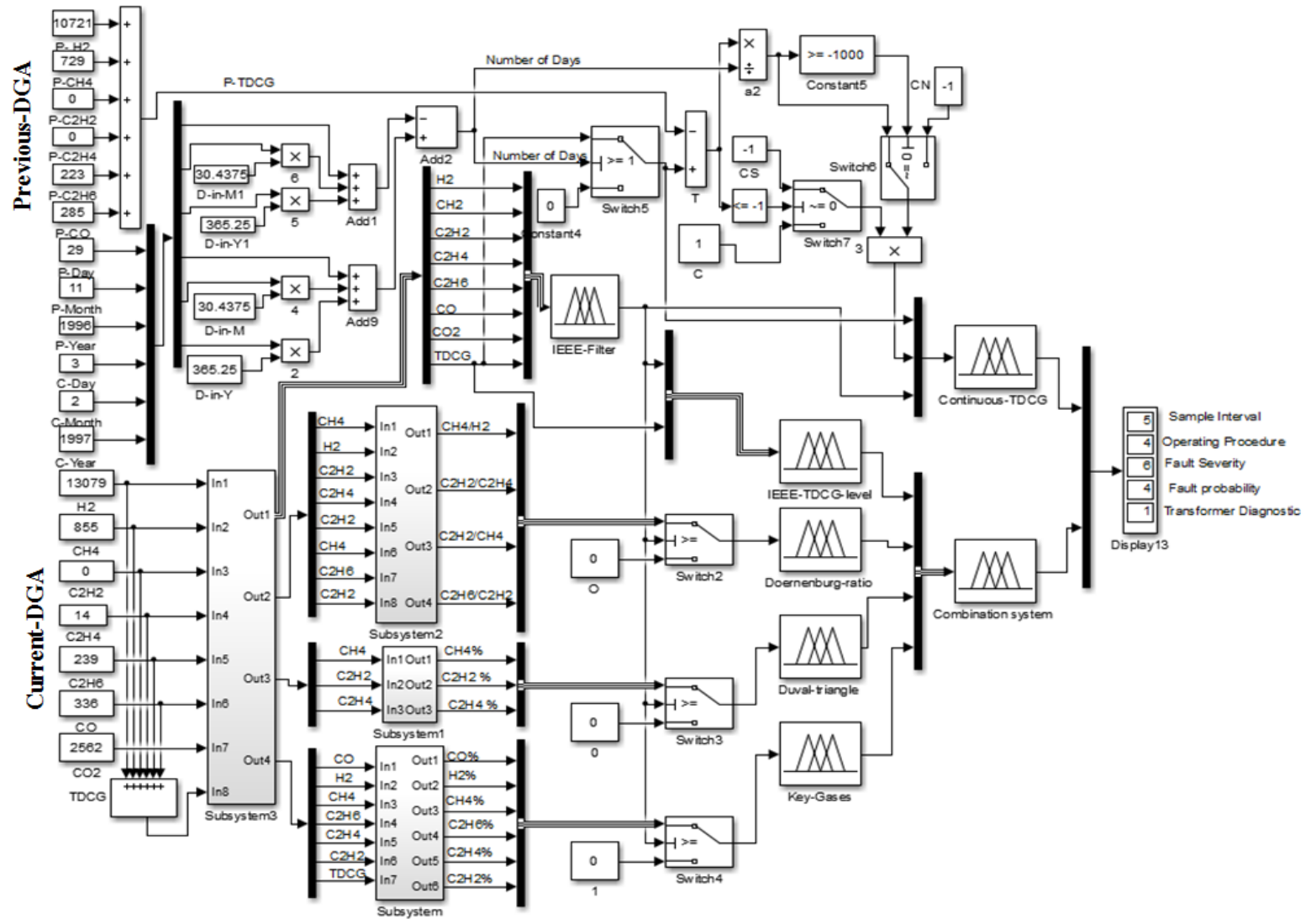

Figure 2: Matlab-Simulink schematic of the proposed system 


\section{SYSTEM VALIDATION FOR CASES With KNOWN FAULTS}

To examine the accuracy of the proposed fuzzy-based diagnostic system, data from a number of sources have been assessed. References, i.e. [3], [8], [10] and [15] provided 444 DGA results, including well characterized diagnostics of the transformers reported. As will be shown in this section, the different DGA interpretation techniques proposed in International Standards do not always lead to the same conclusions regarding a transformer fault. For that reason it is important being able to combine different approaches in order to provide a more reliable diagnosis.

\section{A. IEEE Standard for Assessing Transformer Health}

As discussed, an oil sample's DGA is initially tested using an IEEE-Filter to identify its normality and abnormality. When 7-key gases and TDCG levels are within a threshold, a transformer is classified as normal, i.e. "healthy". In case any single gas level/ratio surpasses the normal limit, the transformer will be classified to be "unhealthy". Data for all transformers, whether indicated as healthy or unhealthy, are tested using the TDCG-level module to identify fault probability.

In 440 of the 444 transformers investigated, the IEEEFilter module output matches the actual reported fault: 37 indicated Regular Operation (healthy) and 403 cases indicated Abnormal Operation (unhealthy).

The TDCG-level module classifies fault probability for both healthy and unhealthy transformers. Table III shows the system assigned healthy cases a very low probability of a fault.

TABLE III. IEEE FILTER AND FAULT PROBABILITY

\begin{tabular}{|c|c|c|c|c|c|c|}
\hline Agree & Disagree & Healthy & \multicolumn{4}{|c|}{ Unhealthy } \\
\hline \multirow{3}{*}{440} & \multirow{3}{*}{4} & 37 & \multicolumn{4}{|c|}{403} \\
\cline { 3 - 7 } & 4 & \multicolumn{6}{|c|}{ Fault Probability } \\
\cline { 3 - 7 } & & Very & Low & Medium & High & $\begin{array}{c}\text { Very } \\
\text { High }\end{array}$ \\
\cline { 3 - 7 } & & 37 & 145 & 104 & 59 & 95 \\
\hline $99.10 \%$ & $0.90 \%$ & $8.33 \%$ & $32.66 \%$ & $23.42 \%$ & $13.29 \%$ & $21.40 \%$ \\
\hline
\end{tabular}

When Abnormal Operation is indicated, fault probability is increasing. Of the 403 transformers classified as abnormal, 145 transformers are classified as abnormal with low fault probability as the TDCG is within normal limits while having one or more key gases slightly exceeding normal limits.

Of the 403 transformers classified as abnormal, 145 transformers are classified as abnormal with low fault probability as the TDCG is within normal limits while having one or more key gases slightly exceeding normal limits.

\section{1) Accuracy of Individual Diagnostic Method}

For transformers classified as unhealthy, the developed system applies three individual fuzzy logic interpretation algorithms to identify the fault type. The fault indicated by each module is compared to the fault reported in the source document and the accuracy of each algorithm is evaluated. As can be seen in Table IV, Duval is the most accurate technique although Doernenburg also does well. These findings were taken into account during the development of the rules for the combined module that states the type of fault.
TABLE IV. ACCURACY OF INDIVIDUAL TECHNIQUES

\begin{tabular}{|c|c|c|c|c|}
\hline Method & $\begin{array}{c}\text { Number of samples } \\
\text { with known faults }\end{array}$ & $\mathbf{x}$ & $\sqrt{ }$ & Accuracy \\
\hline Duval Triangle & \multirow{2}{*}{403} & 14 & 389 & $96.52 \%$ \\
\hline Doernenburg ratio & & 17 & 386 & $95.78 \%$ \\
\hline \multicolumn{2}{|c|}{ Key Gases } & 75 & 328 & $81.39 \%$ \\
\hline $\begin{array}{l}\text { For all subsequent tables, } \\
\text { x indicates disagreement between known fault and diagnosis, } \\
\sqrt{ } \text { indicates agreement between known fault and diagnosis. }\end{array}$ \\
\hline
\end{tabular}

2) Agreement Among the Three Techniques

Subsequent to the analysis in Table IV, where the agreement of individual techniques to particular case histories is indicated, the agreement and disagreement among the three techniques and to the reported fault was investigated. This analysis was used to develop a set of rules for the combination approach in order to improve the approach overall decision accuracy.

Table $\mathrm{V}$ shows analysis of the matching and non-matching indication by the three techniques. For over $76 \%$ of cases all 3 techniques agree with the reported fault. Almost $22 \%$ of cases had 2 techniques agreeing with the reported fault, i.e. rows 2, 3 and 4 , in such cases the indication of agreement by two methods is considered by the combination module. In cases where no agreement exists, rows 5 to 8 , the proposed system uses Duval indication as the overall decision, as this had previously been shown to be the most accurate technique.

TABLE V. Detailed agreement Percentages for Reported Faults

\begin{tabular}{|c|c|c|c|c|}
\hline Duval & Doernenburg & Key-Gases & No. Samples & $\mathbf{\%}$ \\
\hline 1 & 1 & 1 & 307 & $76.18 \%$ \\
\hline 1 & 1 & 0 & 70 & $17.37 \%$ \\
\hline 0 & 1 & 1 & 9 & $2.24 \%$ \\
\hline 1 & 0 & 1 & 9 & $2.24 \%$ \\
\hline 0 & 1 & 0 & 0 & $0 \%$ \\
\hline 1 & 0 & 0 & 3 & $0.74 \%$ \\
\hline 0 & 0 & 1 & 3 & $0.74 \%$ \\
\hline 0 & 0 & 0 & 2 & $0.49 \%$ \\
\hline & & & 403 & $100 \%$ \\
\hline
\end{tabular}

- Combination rules

For transformers which had been identified as being faulty, as shown in Figure 1, the Duval Triangle, Doernenburg ratio and Key gas method modules are automatically operated to individually determine the fault type. Thereafter, the three variable output codes are entered into the combination system, based on results presented in Tables IV and $\mathrm{V}$, to improve the accuracy of diagnosis. As shown in Figure 1, where the key indicators $\mathrm{A}, \mathrm{B}$ and $\mathrm{C}$ relate to number of techniques in agreement, the developed module indicates the relevant fault types as classified in Table I.

\section{3) Overall accuracy of the proposed system}

Using 403 faulty transformers' data, the proposed system has over $98.7 \%$ success in indicating faults that match reported faults. This provides over 3\% improvement on identifying faults in comparison to individual techniques under study.

\section{Discussion OF THE CASES STUdied}

Table VI presents the reported fault and the corresponding diagnostic cases for 4 sample transformers. These cases have been chosen to demonstrate some samples of the module outputs of the recommended approach, i.e. the IEEE-Filter indication, the agreement and disagreement of the interpretation techniques and different fault probabilities. The IEEE-Filter module indicates normality condition, IEEE-TDCG module identifies their contents level, Doernenburg, Duval and Key gas are indicators for faulty 
transformers and the overall decision of the algorithm indicates the state of the transformer and fault probability.

TABLE VI. Selected CASES of THE VALidation Process

\begin{tabular}{|c|c|c|c|c|c|c|c|c|c|}
\hline No & Rf & IEEE-Filter & $\begin{array}{c}\text { IEEE-TDCG } \\
\text { Level }\end{array}$ & Doernenburg & Duval & Key gas & Oviagnosis & $\begin{array}{c}\text { Fault } \\
\text { probability }\end{array}$ & Fault \\
\hline 1 & {$[16]$} & Normal & Very low & Off & Off & Un-defined & Normal & Very low & Normal \\
\hline 2 & {$[17]$} & Ab-Normal & Medium & PD & T1 & PD & PD & Medium & PD \\
\hline 3 & {$[18]$} & Ab-Normal & Very high & Thermal & T1 & Thermal in oil & T1 & Very high & Over Heat \\
\hline 4 & {$[19]$} & Ab-Normal & Low & Thermal & T2 & Thermal in oil & T2 & Low & LTF \\
\hline
\end{tabular}

In samples 2, 3 and 4, based on IEEE-Filter outcome, (C$\mathrm{S}$ is Abnormal) and the three transformers are classified as unhealthy. The 3 interpretation modules operate in parallel with the IEEE-TDCG-level, determining the fault type and probability. For sample 2 Doernenburg and Key gas modules indicate that a PD fault exists, however Duval indicates T1, the combination rules for the overall decision consider the two agreeing modules. Samples 3 and 4 show good correlation of the thermal fault indicated by the system and by written reports

\section{A. Analysing Non-Matching indications}

There were two sets of non-matching results between the reported fault and output diagnostic tool.

In the first set there was a difference between the prediction of IEEE-Filter and the actual diagnosis. As discussed in the section dealing with the IEEE-filter, in Table III, there were 4 samples in which the output did not match the reported fault.

The second set, containing 5 samples, is identified after running the interpretation techniques: there is a difference between the approach overall decision and the reported fault.

\section{B. Analysing Non-Matching indications}

There were two sets of non-matching results between the reported fault and output diagnostic tool.

In the first set there was a difference between the prediction of IEEE-Filter and the actual diagnosis. As discussed in the section dealing with the IEEE-filter, in Table III, there were 4 samples in which the output did not match the reported fault.

The second set, containing 5 samples, is identified after running the interpretation techniques: there is a difference between the overall decision of the system and the reported fault.

\section{ApPliCAtion OF THETRENDING Diagnostics MODUle}

A major advantage of this system is realized in its ability to apply the standards, based on current and previous samples, to provide full information of the transformer state for continuing operation. Samples of regularly monitored transformers, collected from published articles, i.e. [15] and [21] are used to validate the proposed trending algorithm. As explained earlier, the Trending TDCG module operates on data from consecutive sample times and provides information on fault severity, recommends sampling interval and operating procedure. To evaluate the trending module, 134 DGA samples for 11 tested transformers with different power ratings are considered.
As a result of testing these transformers, using available previous/current DGA data, it can be observed that the module has the ability to provide full information of the transformer health during operation and can also detect changes in condition by comparing the current and previous analyses. The trending of the DGA history provides an ongoing classification for the fault severity; recommend sampling intervals and future operating procedure.

To outline operation, 10 samples from 1 transformer are considered in detail as follows;

\section{A. Case study:}

The data for this case, shown in Table VII, analysis is presented in Figure 3. System output for sample 1 shows a T2 fault with low fault probability. Samples 2 and 3: continue to report T2 with low probability, suggesting the transformer has not been repaired: fault severity is very low, annual sampling advised and continues regular operation.

Samples 4 and 5: T2 fault continues to be reported but the indications and recommendations become stronger.

TABLE VII. DATA FOR A TRANSFORMER REPORTED IN [20]

\begin{tabular}{|c|c|c|c|c|c|c|c|}
\hline Sample & Date & $\mathbf{H}_{\mathbf{2}}$ & $\mathbf{C H}_{\mathbf{4}}$ & $\mathbf{C}_{\mathbf{2}} \mathbf{H}_{\mathbf{2}}$ & $\mathbf{C}_{\mathbf{2}} \mathbf{H}_{\mathbf{4}}$ & $\mathbf{C}_{\mathbf{2}} \mathbf{H}_{\mathbf{6}}$ & $\mathbf{C O}$ \\
\hline 1 & $15 / 09 / 1995$ & 3 & 19 & 0 & 9 & 4 & 539 \\
2 & $18 / 09 / 1996$ & 0 & 20 & 0 & 13 & 9 & 467 \\
3 & $09 / 05 / 1997$ & 0 & 30 & 0 & 9 & 3 & 578 \\
4 & $27 / 08 / 1998$ & 26 & 54 & 0 & 22 & 10 & 942 \\
5 & $12 / 04 / 1999$ & 21 & 60 & 0 & 28 & 6 & 731 \\
6 & $10 / 09 / 2002$ & 305 & 648 & 0 & 691 & 192 & 657 \\
7 & $15 / 10 / 2002$ & 569 & 1364 & 7 & 1703 & 451 & 552 \\
8 & $22 / 10 / 2002$ & 573 & 1637 & 6 & 1965 & 520 & 643 \\
9 & $28 / 10 / 2002$ & 227 & 1616 & 7 & 2002 & 535 & 599 \\
10 & $10 / 12 / 2002$ & 1 & 22 & 0 & 7 & 6 & 5 \\
\hline
\end{tabular}

Sample 6: fault changes from $\mathrm{T} 2$ to $\mathrm{T} 3$, the fault probability, severity and operating procedures step up. The presence of an alarm indicates that the transformer has become a critical case

Dates for samples 7,8 and 9 indicate that the sample interval was shortened, suggesting operators know of the fault.

Over this period the probability of $\mathrm{T} 3$ has increased from high to very high with excessive fault severity, advised daily sampling and removal from service.

The dissolved gas levels are reduced in sample 10; this indicates maintenance activity, confirmed by the maintenance history data [20].

Figure 3 shows the approach's result based on the historical DGA data for the running period, it should be noted that sample 10 is not included in the figure as the module would be restarted due to the maintenance activities.

It is clear from the results that the proposed approach is indicating the health of the transformer correctly and 
suggesting the right sampling intervals to keep the transformer operating safely.

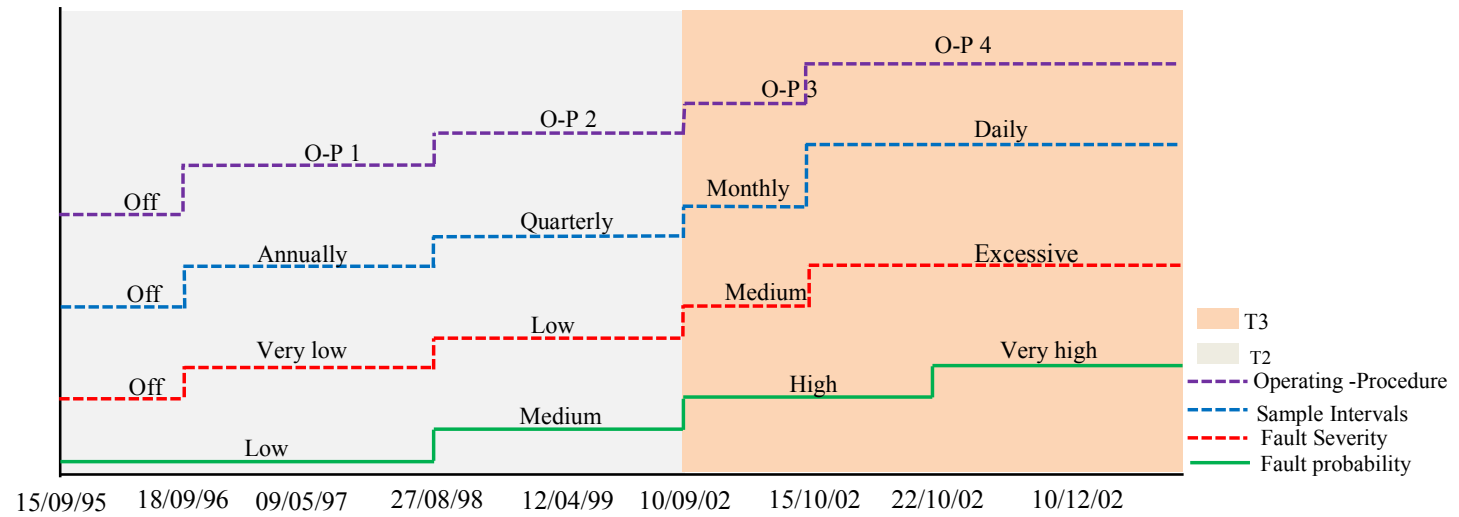

Figure 3: The approach outcomes for on-going condition monitoring of a tested transformer

\section{CONCLUSION}

In this work, multiple fuzzy logic modules of the proposed diagnosis system for continuous condition monitoring of power transformers were developed. Fuzzy sets and rules were formulated for three Dissolved Gas Analysis interpretation methods, further these were combined into one augmented module in order to increase the accuracy of fault identification, fault probability, fault severity, futuristic sampling intervals and recommended operating procedure. The fuzzy system is demonstrated to present a satisfactory performance in the identification of the transformer faults and future recommendation.

The proposed system has been validated using DGA data from published articles. The accuracy of the individual diagnostic techniques, namely Duval triangle, Doernenburg ratio and Key gas, is increased when the augmented combination algorithm is used.

The proposed system is proved to be highly capable of classifying the normality and abnormality of the transformer and its fault probability. This has been confirmed and validated through 1290 transformer related DG samples. Moreover, the proposed approach was accurately able to identify type of the fault and providing the final decision based on the combination of 3 interpretation techniques.

The proposed system was successfully applied to trending of power transformer DGA data. The system output provides accurate information on fault severity, sample interval and recommends an operating procedure for the transformer, based on the TDCG current level and daily increment rate recommendations within IEEE C57.140.

The accuracy of the system has been found to be higher than that of any of the individual analysis, and its application could lead to a more reliable diagnosis of transformers.

\section{REFERENCES}

[1] M. Beykverdi, F. Faghihi, and A. Moarefian, "A New Approach for Transformer Incipient Fault Diagnosis Based on Dissolved Gas Analysis ( DGA )," Nov. J. Eng. Appl. Sci., vol. 2, pp. 1-7, March 2014.

[2] A. Ashkezari, T. Saha, C. Ekanayake, and H. Ma, "Evaluating the accuracy of different DGA techniques for improving the transformer oil quality interpretation," 21st Australas. Univ. Power Eng. Conf., pp. $1-6,2011$.

[3] H. Nabwey, E. Rady, A. Kozae, and A. Ebady, "Fault Diagnosis of Power Transformer Based on Fuzzy Logic Rough Set theory and Inclusion Degree Theory," online J. Power Energy Eng., vol. (1), no. 2, pp. 45-49, 2008.
[4] R. Hooshmand and M. Banejad, "Application of Fuzzy Logic in Fault Diagnosis in Transformers using Dissolved Gas based on Different Standards," World Acad. Sci. Eng. Technol., no. 17, pp. 157-161, 2007.

[5] H. Malik, T. Mahto, B. Anil, K. Mantosh, and R. Jarial, "Fuzzy-logic applications in transformer diagnosis using individual and total dissolved key gas concentrations," J. Electr. Eng., vol. 12, no. 3, pp. 105-109, 2012

[6] L. Decun and W. Guochun, "Global fault diagnosis method of traction transformer based on Improved Fuzzy Cellular Neural Network," 4th IEEE Conf. Ind. Electron. Appl., pp. 353-357, 2009.

[7] R. Naresh, V. Sharma, and M. Vashisth, "An Integrated Neural Fuzzy Approach for Fault Diagnosis of Transformers," IEEE Trans. Power Deliv, vol. 23, no. 4, pp. 2017-2024, 2008.

[8] R. Hooshmand, M. Parastegari, and Z. Forghani, "Adaptive NeuroFuzzy Inference System Approach for Simultaneous Diagnosis of the Type and Location of Faults," IEEE Electr. Insul. Mag., pp. 3242, 2012.

[9] I. DA Silva, M. Imamura, and A. De Souza, "The application of neural networks to the analysis of dissolved $\backslash$ ngases in insulating oil used in transformers," in Smc IEEE conf on power systems, vol. 4, pp,. 2000,

[10] A. Akbari, A. Setayeshmehr, H. Borsi, , and I. Fofana, "Intelligent agent-based system using dissolved gas analysis to detect incipient faults in power transformers," Electr. Insul. Mag. IEEE, vol. 26, no. 6. pp. $27-40,2010$

[11] Y. Tu and Z. Qian, "DGA Based Insulation Diagnosis of Power Transformer via," in the 6th International Conference on Properties and Applications of Dilectric Materials, pp. 133-136. 2000.

[12] "IEEE Std C57.104-2008 - IEEE Guide for the Interpretation of Gases Generated in oil-Immersed Transformer," IEEE Power Energy Soc. Spons. by Transform. Comm., no. 2, pp. 1-36, 2009.

[13] "Transformer Diagnostics," Facil. Instr. Stand. Tech. United States Depsrtment Inter. Bur. Reclamation, Spons. by Hydroelectr. Res. Tech. Serv. Gr., vol. 3-31, pp. 1-63, 2003.

[14] M. Duval, "The Duval Triangle for Load Tap Changers, NonMineral oils and Low Temperature Faults in Transformers," IEEE Electr. Insul. Mag., vol. 24, no. 6, pp. 22-29, 2008.

[15] D. R. Morais, J. R. d. Silva, and J. G. Rolim, "A Fuzzy System for Detection of Incipient Faults in Transformers based on the Dissolved Gas Analysis of Insulating Oil," Diagnostics Electr. Mach. Power Electron. Drives, SDEMPED. 5th IEEE Int. Symp., vol. 21, no. 2, pp. $1-6,2005$.

[16] A. Abu-Siada, S. Hmood, and S. Islam, "A new fuzzy logic approach for consistent interpretation of dissolved gas-in-oil analysis," IEEE Trans. Dielectr. Electr. Insul., vol. 20, no. 6, pp. 2343-2349, 2013.

[17] S. S. M. Ghoneim and I. B. Taha, "Artificial Neural Networks for Power Transformers Fault Diagnosis Based on IEC Code Using Dissolved Gas Analysis," Int. J. Control. Autom. Syst., vol. 4, no. 2, pp. $18-21,2015$

[18] H. Ikbal and S. Farag, "Monitoring Power Transformer Using Fuzzy Logic," J. Eng. Dev., vol. 17, no. 6, pp. 146-163, 2013.

[19] H. V Waghmare and P. H. Kulkarni, "Modeling of Transformer DGA using IEC \& Fuzzy Based Three Gas Ratio Method," Int. J. Eng. Res. Technol. IJERT, vol. 3, no. 9, pp. 1149-1152, 2014. 
[20] J. Pathak, and J. McCalley, "Condition data aggregation with application to failure rate calculation of power transformers," IEEE. Proc. 39th Annu. Hawaii Int. Conf. Syst. Sci., vol. 10, pp. 1-10, 2005.

[21] S. Ghoneim and K. Shoush, "Diagnostic Tool for Transformer Fault Detection Based on Dissolved Gas Analysis," IOSR J. Electr. Electron. Eng. www.iosrjournals.org, vol. 9, no. 5, pp. 20-26, 2014. 